16 | 1994

Varia

\title{
Ars discendi, ars docendi : programme Pascal
}

Thérèse Goyet

\section{OpenEdition}

\section{Journals}

Édition électronique

URL : http://journals.openedition.org/ccibp/592

DOI : $10.4000 /$ ccibp.592

ISSN : 2493-7460

\section{Éditeur}

Centre international Blaise Pascal

\section{Édition imprimée}

Date de publication : 5 avril 1994

Pagination : 31-34

ISSN : 0249-6674

\section{Référence électronique}

Thérèse Goyet, «Ars discendi, ars docendi : programme Pascal », Courrier du Centre international Blaise Pascal [En ligne], 16 | 1994, mis en ligne le 07 janvier 2016, consulté le 19 avril 2019. URL : http:// journals.openedition.org/ccibp/592 ; DOI : 10.4000/ccibp.592

Ce document a été généré automatiquement le 19 avril 2019.

Centre international Blaise Pascal 


\title{
Ars discendi, ars docendi : programme Pascal
}

\author{
Thérèse Goyet
}

1 Le latin de l'intitulé, c'est pour le plaisir, mais on peut lui donner quelques autres justifications. Ars évoque tout à la fois le savoir-faire, le talent, la compétence professionnelle, le goût de la solution élégante. La parataxe permet le va-et-vient entre le thème et le prédicat (sujet et attribut). On interprétera au choix : savoir étudier, c'est savoir enseigner. Ou : pour apprendre, il faut enseigner; ou : l'enseignement consiste en étude ; et d'autres variations communes sur une vérité que l'expérience méditée permet seule de comprendre. Car on passe de l'état de discipulus à celui de doctus ou même doctor moyennant quelques épreuves (par exemple, le concours d'agrégation) mais sans discontinuité, et vice-versa.

2 Quant à la ponctuation des deux points qui présente le terme : " Programme Pascal », elle signifie que nous étudions les Pensées de Pascal, et elle voudrait insinuer - chacun en soi-même citera le Mystère de Jésus - que Pascal est l'exemplaire de l'homme en recherche que la vérité nourrit et affame en même temps. (J'enseigne, donc j'apprends).

Pascal au programme: quelle présence dans une vie! que d'occasions dans la carrière d'un professeur de lettres. J'ai pu dire en 1984 que je célébrais les noces d'or de notre rencontre. Aujourd'hui, ce sont les noces de diamant. Pour ce qui est de ma carrière, il est venu la première année, au Lycée Pasteur de Besançon, où je faisais un cours de littérature en classe de terminale. Les élèves m'ont demandé de leur présenter le Mystère de Jésus. De ce don je leur suis pour toujours reconnaissante. Les programmes du second degré et de l'enseignement supérieur m’ont ramenée à son œuvre bien des fois. En arrivant à Clermont à la Faculté des Lettres, en 1965, j'ai reçu le patrimoine Pascal comme un héritage sans demander le bénéfice de l'inventaire. J'ai donc pris en charge l'organisation de travaux collectifs. En 1970, c'était le tricentenaire du livre des Pensées. En 1976, coïncidant avec un programme d'agrégation. ce fut un colloque considérable, Méthodes chez Pascal, dont les Actes constituent un volume de 544 pages, avec quarante-quatre communications. Le mot de la fin, Jean Mesnard l'avait eu : «Peut-être un jour aurons-nous envie de recommencer. » 
C'est ce qui est arrivé. Nous avons fondé, pour l'information, la documentation et la concertation scientifique, sur le riche terrain de la Bibliothèque municipale et interuniversitaire, le Centre international Blaise Pascal. L'Université de l'Auvergne s'est enfin mise sous l'intitulé Blaise Pascal. Et c'est ainsi que nous sommes rassemblés aujourd'hui en vue d'ameublir un texte du programme d'agrégation, par l'accroissement de ferveur et de lumière que dégage la communication, quand elle est motivée en soi, et effectivement amicale.

\section{Pascal « appliqué »}

5 Notre journée, pédagogique et scientifique, renouvelle un colloque de même inspiration que nous avons tenu au Centre régional de Documentation pédagogique en deux sessions, novembre 1984 et mai 1985. Des «journées de réflexion» avaient rassemblé des spécialistes de la recherche pascalienne et des praticiens de la pédagogie. Les autorités académiques avaient encouragé cette initiative de «recyclage» et de participation. "L'accès aux Pensées de Pascal » s'engageait sur le principe qu'en tout point de recherche on doit simultanément envisager les moyens de l'explication publique. À ceux que décourageraient les difficultés actuelles de l'institution scolaire, des collègues chevronnés démontrent qu'on ne perd rien à "préférer aux écrivains flatteurs les écrivains formateurs" - selon l'expression de Philippe Sellier - et notre vieil ami du Japon, Genji Yasui, raconte comment dans une totale opacité (langue, culture et préoccupations divergentes) des «expériences pascaliennes » peuvent tracer le sillon d'une féconde inquiétude.

6 Les actes de ce colloque viennent seulement de paraître (Klincksieck, 1993). Ce long délai a entraîné quelques modifications, dans le contenu et dans la structure. Notre premier dessein se proposait une petite bibliographie, des livres indispensables, ou très commodes, selon la situation du marché du livre d'enseignement. Mais il nous est apparu que cette sorte d'appareil serait vite périmé, d'ailleurs incertain entre le trop pour ceux dont le temps et les moyens d'accès sont limités, et le trop peu pour les lecteurs entrânées. Nous avons donc préféré la note bibliographique de justification en circonstance, ce qui nous permet de faire entrevoir de nombreuses pistes possibles.

7 Nous avons pris le temps de réunir une illustration qui serait poétique autant qu'historique, afin d'harmoniser le vécu de Pascal avec le nôtre. Et nous avons cherché la commodité en vue de travaux pratiques. L'écriture de Pascal, par exemple, est donnée d'après les meilleures photographies, celles que la Bibliothèque nationale a récemment tirées " par contact ». Des pages entières sont présentées sur papier glacé, et, pour les espaces découpés sur papier ordinaire, l'imprimeur a pris grand soin de placer la transcription en regard. L'examen à la loupe ou la projection sur écran sont évidemment souhaitables, mais si l'on prend soin de préparer la lecture à deux voix et de l'enregistrer à l'avance, il nous semble qu'une attention moyennement soutenue peut permettre à nos élèves de suivre le mouvement de la plume de Pascal et de capter ainsi un peu de sa respiration créatrice. Un audio-visuel actif propose ainsi une voie de découverte facilitée.

La convergence des « grandes avenues » et des " chemins qui marchent » ne fut pas entre nous un tracé commandé abstraitement par un mot d'ordre. C'est donc la mobilité qui est demandée au lecteur afin qu'il se place tantôt à des points de vue dominants, tantôt en des points de percée particuliers à nos guides. On trouvera dans ces Actes: la biographie 
de Pascal en tant qu'elle s'incorpore à son œuvre; son contexte théologique ; l'histoire du livre se fabriquant selon la succession des mentalités ; une saisie du style à travers les éléments de son vocabulaire et dans ses mouvements; des thèmes révélateurs où le moi de Pascal s'exprime ou se dépasse, et une idée de sa répercussion chez des écrivains de date récente. Les discussions qui purent être reproduites ont gardé l'animation des propos sur «les entretiens ordinaires de la vie». Enfin une série de témoignages pédagogiques nous apprend des moyens de patience audacieuse par lesquels on peut ouvrir Pascal à des écoliers qui, généralement, n'y pensaient guère.

Pris comme compagnon actif du métier de l'enseignement, ces Actes culminent en efficacité avec la "péroraison» que Dominique Descotes présente par un slogan de publicité : Do it yourself: Oui, faites dire Pascal à vos élèves. Nul style n'est plus capable de les faire progresser dans l'ars dicendi dont nous sommes les serviteurs. Et l'appel à la mémoire force le diseur à toucher la pensée. Sur une scène on devient responsable du texte qu'on a en la bouche. L'intonation propre issue de la générosité de l'acteur lui revient et le marque lui-même. L'auditeur, lui, pourra en conserver l'atteinte pendant des années, comme nous l'avons éprouvé.

(On trouvera dans les discussions au cours du livre un débat sur la «gustation» des textes, à concerter avec leur maniement thématique).

11 Par définition la table des matières a la fonction de guide. Toutefois nous détacherons dans ce livre deux articles dont l'utilité nous semble exceptionnelle en raison de leur altitude de pensée et par la précision de leur écriture. À savoir "l'ouverture» existentielle et la situation théologique. A tous deux je décernerai le plus grand de mes éloges en disant qu'ils ont atteint la simplicité. Et j'ose déclarer que c'est ma fierté d'avoir suscité et publié ces deux contributions de la part des deux seuls maitres qui pouvaient les produire.

12 En toutes ses occasions Pascal "appliqué » aboutit à un Pascal "expliqué ». Une explication qu'on trouvera en plusieurs lieux dans ce livre, c'est la raison du changement des éditions les plus en usage dans l'Université. J'en ai vécu... l'application. L'édition des Pensées régnante dans ma jeunesse, c'était celle de Léon Brunschvicg. Elle plaît aux écrivains qui cherchent dans Pascal une motion, ou l'émotion, parce qu'on y retrouve facilement les morceaux qu'on a élus. (Voir l'article de Michel Lioure.) Après un court passage dans l'édition de Jacques Chevalier, j'ai été attachée pendant des années assez nombreuses, à l'édition Lafuma publiée au Seuil (édition sur laquelle a été faite la Concordance Davidson-Dubé dont on vous parle souvent. Édition " objective " puisqu'elle est tirée, quant à son ordonnance, d'un objet incontestablement constitué, c'est-à-dire une copie issue des éditeurs de Port-Royal. L'édition à votre programme se réfère à une autre copie d'origine plus familiale. Livre soigneusement imprimé, solidement broché, très utilement annoté. Lecteurs jeunes, vous n'avez peut-être pas connu d'autre structure: vous n'aurez donc pas à accommoder votre regard ni à transposer de numérotation. Cependant je voudrais vous dire un mot sur les raisons de ces mutations.

13 Vers 1970, j'ai été curieuse de savoir comment Port-Royal, dans sa révérence passionnée, avait pu exprimer les volontés de Pascal, tout en s'accordant l'usage des ciseaux et les atténuations du style. J'ai élaboré une correspondance numérique entre la première édition et les éditions Lafuma et Brunschvicg, la plus grande difficulté résidant en ce que la délimitation des fragments n'est pas la même ${ }^{1}$. J'ai alors pensé que, parmi les causes de son succès, l'édition de Port-Royal avait eu sa commodité. D'abord les «Pensées sur la religion» montrent une construction autonome d'apologie; les Pensées «sur quelques 
autres sujets » se disposant après, en dehors du plan démonstratif, et elles ont une coloration d'ensemble laïque, les réflexions profanes étant coupées de chapitres théologiques qui s'intègrent peu dans le déroulement (à partir du chapitre XXI, jusqu'au dernier, XXXI, «Pensées diverses »), une prière scellant la fermeture du tout. L'édition Brunschvicg, elle, procède par logique analytique, les pensées laïques étant placées au commencement. En composition inversée, ces deux systèmes ont un effet commun : c'est la nature intellectuelle de la filière organisatrice. Nous nous apercevions que les port-royalistes d'époque cartésienne, inféodés à leur théologie, et le grand rationaliste Brunschvicg, se soumettent la conduite de l'esprit de Pascal par le fait de distribuer ses morceaux selon les besoins propres de leurs raisons respectives.

Aujourd'hui... un parcours géométrisé nous intéresse moins. Notre ambition serait plutôt de prier Pascal de nous laisser approcher pas à pas et de reprendre à volonté la conversation avec lui. Sur le modèle grammatical Doceo pueros grammaticam si j'ose risquer un Doce juvenes Pascalem, ce sera selon un ordre du sentiment et sous l'influence multiforme dont je suis enveloppée. Pour conduire cette approche des sensibilités, on demande à Pascal d'être notre pédagogue, c'est-à-dire le conseiller vigoureux, rigoureux, et cependant intérieurement accordé à qui l'écoute.

\section{La leçon pascalienne}

$15 \mathrm{Au}$ fond de toutes les « preuves » que Pascal met en marche concertée à la découverte du " Dieu caché ", se trouve affirmé le caractère historique de la Révélation. Seulement la conception de l'historicité qu'il partage avec l'ensemble de ses contemporains, parait bien étroite. Le monde alors n'avait que quatre mille ans! «Sem, qui a vu Lamech, qui a vu $\mathrm{Adam}^{2}$, etc. » Une si heureuse passation insère dans l'enchaînement providentiel un maillon fragile. Et quand Pascal, ayant entrepris auprès de ses amis « de prouver la vérité de la religion par les prophéties [...], il les expliqua d'une manière fort intelligible, il en fit voir le sens et la suite avec une facilité merveilleuse ${ }^{3}$ ", cette clarification trop réussie nous paraît spécieuse. La science exégétique de Pascal est attestée, mais nous nous rappelons que les intellectuels de son temps ignoraient l'ampleur des connexions et de l'histoire et de l'Écriture. Cela serait dommageable pour notre disposition à la confiance, si Pascal tenait ses explications pour des motifs de sa foi. Ce ne sont que des accès, entre autres accès, offerts à l'intelligence: celle-ci est sommée d'aborder les confins du mystère :

La foi embrasse plusieurs vérités qui semblent se contredire.

[...]

La source en est l'union des deux natures en Jésus-Christ. ${ }^{4}$

Voilà donc le disciple de Pascal remis au travail'5. Mais sombrera-t-il dans l'effroi si Pascal le prévient qu'en sortant de ce monde il peut tomber «dans les mains d'un Dieu irrité ${ }^{6}$ ? Enfin de quel droit ce qualificatif pour un maître bon?

17 Certes Pascal adhère à un système théologique, et dans sa place forte tout le monde n'est pas convié. On trouve que le système du jansénisme manque des vérités intermédiaires. Les heurts entre les nécessités (grâce, liberté, etc.) nourrissent donc un état de tension. Par l'effet de la doctrine, ou de sa volonté, ou de son tempérament, la violence de Pascal éclate comme issue d'un instinct vital. C'est une urgence: il faut réveiller « une si extravagante créature, qui vit en somnambule et qui en fait vanité. » 
Ce repos dans cette ignorance est une chose monstrueuse et dont il faut faire sentir l'extravagance et la stupidité à ceux qui y passent leur vie, en la leur représentant à eux-mêmes pour les confondre par la vue de leur folie ${ }^{7}$.

Si le veilleur ne criait pas, il trahirait la cité.

S'il arrive qu'il se fasse entendre, c'est qu'un sentiment naturel, un instinct, veillant en nous, lui correspond. L'ennui attaché à la condition des hommes prépare le terrain : «Ils sentent alors leur néant sans le connaître ${ }^{8}$.» C'est "par la nature même ${ }^{9}$ » que Pascal amène une telle créature à introduire son propre procès. Au chapitre de la « Transition de la connaissance de l'homme à Dieu ", c'est sur l'appui (si l'on peut dire) de la connaissance naturelle, ou science physique, que se déclenche l'ouverture spirituelle: "Que l'homme considère ce qu'il est au prix de ce qui est ${ }^{10}$.» L'incompréhensibilité cosmique jouxtera dans l'esprit ébranlé l'incompréhensible de l'existence divine. Nous voici projetés dans l'évidence de notre incompétence foncière, et la déception est sanglante au sujet du bonheur promis par les philosophes : «Et cela n'est pas vrai. [...] Et cela n'est pas vrai ${ }^{11}$.»

La recherche individualiste de Dieu est tout aussi accablante puisque «il y a une opposition invincible entre Dieu et nous, et [...] sans un médiateur il ne peut y avoir de commerce $^{12}$.» Celui qui n'a pas rencontré Jésus-Christ, c'est-à-dire la grâce, est-il condamné au désespoir? et ne ferait-il pas bien de s'abandonner à la paresse et aux plaisirs? On a souvent reproché aux jansénistes d'y engager, par voie de conséquence, les libertins et les indifférents. Et notre société, par ses angoisses comme par ses séductions multiformes, nous incite à la même dénaturation. Mais la grandeur incluse dans notre nature ramène au bon sens, et, au lâche évasif le prophète lance le défi de l'honneur :

Vous auriez bientôt la foi si vous aviez quitté les plaisirs. Or c'est à vous de commencer ${ }^{13}$.

La balle sera donc toujours en quelque façon dans le camp de l'homme.

On peut se demander s'il se trouve du monde pour la ramasser. Entrer dans le jeu selon Pascal, c'est courir le danger de penser à se convertir. Les collègues qui nous ont rapporté les réactions de leurs élèves témoignent que ceux-ci le pressentent fort bien.

Ils auraient donc compris la leçon pascalienne... Comment cela a-t-il pu se faire?

On sait combien le don de communication chez Pascal enchantait son entourage. L'inspiration de la charité a fait fructifier le don. Elle a du même coup écarté la superbe doctorale. Car le plus urgent de la rhétorique, c'est d'« échauffer ", c'est-à-dire d'insinuer le désir d'aimer Dieu, plutôt que d' "instruire ${ }^{14}$ ", c'est-à-dire de contenter la curiosité même la plus légitime. La conversation que Pascal engage fait pour l'interlocuteur un encouragement naïf à sortir de sa honte, à exprimer son fond à son tour. Qu'on ne lui reproche pas comme une complaisance d'artiste à soi-même le soin de sa rédaction. Même si sa volonté d'humilité en a souffert, son devoir était de séduire.

On discutera si son esthétique est baroque : elle est prenante. Par exemple la déclaration de contradiction entre nos deux natures se passe comme un échange sportif.

S'il se vante, je l'abaisse.

S'il s'abaisse, je le vante ${ }^{15}$

6 Spontanément la comédie s'invente avec ces gens « qui font les entendus ${ }^{16}$ ». Il n'y a qu'à s'asseoir dans un fauteuil pour la regarder, ou bien aller dans la rue pour voir les carrosses se dépasser les uns les autres. Quelle allégresse de l'humour! Quel sentiment des différences (entre deux grains de raisin) entraîne les variations de l'allure! Le vocabulaire ne peut se dispenser des termes spéciaux, mais jamais le choix n'est guindé ${ }^{17}$. 
Le spectacle de l'univers ou celui de l'histoire humaine dilate l'imagination mais - et ceci prime dans la conscience du professeur - sans la dérégler. Et quels beaux incendies, allumés par les paroxysmes de Pascal, laissent leurs lueurs dans nos mémoires reconnaissantes.

Qu'on nous permette de dire, en conclusion d'un demi-siècle d'expérience pédagogique, que l'énergie des Pensées, c'est leur style. La gaieté, la politesse, le gardent en vie. Encore une fois, n'importe comment on le prenne, le style c'est l'homme. Un sentiment d'aisance héroïque et heureuse sourd immanquablement de la compagnie de l'homme Pascal.

\section{NOTES}

1. Thérèse Goyet. "Table de concordance entre l'édition des Pensées de 1670 et les éditions Lafuma (Luxembourg) et Brunschvicg", dans Les Pensées de Pascal ont trois cents ans, (Clermont-Ferrand, G. de Bussac, 1971) p. 42-78.

2. Pensées, 327-296-625. Le premier numéro est celui de l'édition Sellier, Classiques Garnier, 1991 éventuellement suivi du chiffre de la page-; le deuxième celui de Lafuma, au Seuil ; le troisième, celui de Brunschvicg.

3. Préface d'Étienne Périer, pour l'édition de 1670.

4. Pensées 614-733-862. Nous renvoyons dans Méthodes chez Pascal, à «La méthode prophétique selon Pascal », particulièrement p. 72-74.

5. D'après Pensées, 38-4-184 : « les philosophes [...] travailleront celui qui les recherche. »

6. Pensées, 684, (p. 477)-427-194.

7. Pensées, 682, (p. 482)-428-195.

8. Ibid., 70-36-174.

9. Ibid., 40-6-60.

10. Ibid., 300-199-72.

11. Ibid., 26-407-465.

12. Ibid., 410-378-470.

13. Ibid., 698, (p. 450)-816-240.

14. Ibid., 329-298-283. Il s'agit de l'ordre de la charité, suivi par Jésus-Christ, saint Paul, saint Augustin. Lafuma lit « abaisser » au lieu d'« échauffer ». En ce cas il s'agit d'incliner à l'humilité.

15. Ibid., 163-130-420.

16. Ibid., 117-83-327.

17. Voir dans L'Accès aux Pensées de Pascal, Christian Meurillon, « Clefs pour le lexique des Pensées ", particulièrement p. 130-134 : «Dans les Pensées : honnêteté et non spécialisation », ceci : «La communication n'est pas sacrifiée à l'approche technique du vrai » (p. 133). 
INDEX

Mots-clés : Pascal, Pensées, étude

Keywords : Pascal, Pensées, study

\section{AUTEUR}

THÉRÈSE GOYET

Premier directeur du CIBP

CERHAC, Université Blaise Pascal 